\title{
Microscopic Molecular Machines Help Bacteriophages to Package Their Genomes and Infect Their Hosts
}

Petr G. Leiman ${ }^{1}$, Andrei Fokine ${ }^{1}$, Paul R. Chipman ${ }^{1}$, Victor A. Kostyuchenko ${ }^{1,2}$, Shuji Kanamaru ${ }^{1,3}$, Fumio Arisaka $^{3}$, Vadim V. Mesyanzhinov ${ }^{2}$, and Michael G. Rossmann ${ }^{1}$

${ }^{1}$ Department of Biological Sciences, Purdue University, 915 W. State Street, West Lafayette, IN 47907-2054, USA.

${ }^{2}$ Laboratory of Molecular Bioengineering, Shemyakin-Ovchinnikov Institute of Bioorganic Chemistry, 16/10 Miklukho-Maklaya Street, 117997 Moscow, Russia.

${ }^{3}$ Department of Life Science, Faculty of Bioscience and Biotechnology, Tokyo Institute of Technology, 4259

Nagatsuta, Midori-ku, Yokohama 226-8501, Japan.

The contractile tail of bacteriophage T4 undergoes major structural transitions when the virus attaches to the host cell surface. When triggered by recognizing a host cell, the baseplate at the distal end of the tail changes from a hexagonal to a star shape. This causes the sheath around the tail tube to contract and the tail tube to protrude from the baseplate and pierce the outer cell membrane and the cell wall, before reaching the inner cell membrane for subsequent viral DNA injection. The structures of the T4 extended tail with its hexagonal baseplate and of the contracted tail with its starshaped baseplate have been determined by combining X-ray crystallography and cryo-electron microscopy, showing that the baseplate proteins move as rigid bodies relative to each other during the conformational transition.

A pseudo atomic resolution structure of the head has also been determined demonstrating a common origin for most tailed phages and possibly also some animal viruses. The DNA packaging motor, powered by the sequential hydrolysis of ATP molecules, is situated at a special pentameric vertex. This motor has been studied in the homologous phi29 tailed phage. 